\title{
Early Visual Processing Deficits in Dysbindin-Associated Schizophrenia
}

\author{
Gary Donohoe, Derek W. Morris, Pierfilippo De Sanctis, Elena Magno, Jennifer L. Montesi, \\ Hugh P. Garavan, lan H. Robertson, Daniel C. Javitt, Michael Gill, Aiden P. Corvin, and John J. Foxe
}

Background: Variation at the dysbindin gene (DTNBP1) has been associated with increased risk for schizophrenia in numerous independent samples and recently with deficits in general and domain-specific cognitive processing. The relationship between dysbindin risk variants and sensory-level deficits in schizophrenia remains to be explored. We investigated P1 performance, a component of early visual processing on which both patients and their relatives show deficits, in carriers and noncarriers of a known dysbindin risk haplotype.

Methods: Event-related potential responses to simple visual isolated-check stimuli were measured using high-density electrical scalp recordings in 26 individuals meeting DSM-IV criteria for schizophrenia, comprising 14 patients who were carriers of the dysbindin risk haplotype and 12 patients who were nonrisk haplotype carriers.

Results: Carriers of the dysbindin risk haplotype demonstrated significantly reduced P1 amplitudes compared with noncarriers. A large effect size of $d=.89$ was calculated for the difference in P1 amplitude over scalp sites where the deficit was maximal.

Conclusions: The P1 deficits associated with a dysbindin risk haplotype previously identified in our sample presents functional confirmation ofi ts deleterious effect on brain activity. Building on evidence of dysbindin's role in higher cognitive function, these early visual processing deficits suggest a generalized role for dysbindin in brain function and is likely to be part of the mechanism by which illness susceptibility is mediated.

Key Words: Dysbindin, EEG, ERP, P1, schizophrenia, sensory processing

S chizophrenia is associated with a generalized pattern of cognitive dysfunction of unknown etiology, involving sensory and cognitive processes. Susceptibility genes may increase risk for the disorder through deleterious effects on these processes. Genetic association and functional studies support involvement of dysbindin (DTNBP1; OMIM 607145) in schizophrenia susceptibility (1). Although functional mutations have yet to be identified, dysbindin risk haplotypes influence cortical expression of the gene (2-4). Understanding of the neurobiological role of dysbindin is limited, but functional studies indicate involvement in presynaptic glutamate release (3-5). This suggests that risk variants may have a synaptic consequence in terms of glutamate availability. Recently, dysbindin risk variants have been associated with poorer performance in higher cognitive domains in controls ( $6-7)$ and with both general and domain-specific deficits (e.g., spatial working memory dysfunction) in schizophrenia $(6,8)$.

The relationship between dysbindin risk variation and sensorylevel deficits in schizophrenia has yet to be explored. This is warranted because dysbindin is expressed throughout the brain, including sensory regions such as the occipital cortex. Early visual

From the Neuropsychiatric Genetics Group (GD, DWM, IHR, MG, APC), Institute of Molecular Medicine, Trinity College Dublin, St. James Hospital, Dublin; The Cognitive Neurophysiology Laboratory (GD, PDS, EM, JLM, HPG, JJF), St. Vincent's Hospital, Fairview; and Trinity College Institute of Neuroscience (GD, EM, HPG, IHR, MG, JJF), School of Psychology, Trinity College, Dublin, Ireland; The Cognitive Neurophysiology Laboratory (PDS, JLM, DCJ, JJF), Nathan S. Kline Institute for Psychiatric Research Program in Cognitive Neuroscience and Schizophrenia, Orangeburg, and Program in Cognitive Neuroscience (DCJ, JJF), Department of Psychology, City College of the City University of New York, New York.

Address reprint requests to Gary Donohoe, D. Clin. Psych., Neuropsychiatric Genetics Group, Institute of Molecular Medicine, Trinity College Dublin, St. James Hospital, Dublin 8, Ireland; E-mail: donoghug@tcd.ie.

Received March 30, 2007; revised July 19, 2007; accepted July 19, 2007. processing deficits have repeatedly been demonstrated in schizophrenia, particularly in the magnocellular system, which is glutamate/NMDA-dependent (9). Because dysbindin is implicated in glutamate/NMDA transmission, early visual processing may be a useful brain function in which to investigate dysbindin's impact.

The P1 visual evoked potential (VEP), which occurs between 75 and $110 \mathrm{msec}$ poststimulus and is recorded over both midline and lateral occipital scalp electrodes, is an especially promising component for investigation in this regard. In a series of studies (10-15), deficits in the P1 component have been repeatedly observed in patients with schizophrenia. This effect is strong, with P1 amplitude in patients less than half the strength of that seen in control subjects. Furthermore, a recent study by Yeap et al. (16) found evidence that non-clinically affected first-degree relatives of patients with schizophrenia also show evidence of reduced P1 amplitudes, establishing a genetic contribution to this deficit as a potential endophenotype for the disorder. Additional strengths of the $\mathrm{P} 1$ component as an endophenotype is that it is straightforward to measure and, as a largely automatic response, is not susceptible to the same motivational issues that later cognitive components such as those to which the P300 are prone (17-18).

We investigated the relationship between P1 performance and a three-marker dysbindin haplotype previously associated with increased risk for schizophrenia in two independent samples, including the sample reported here (19). Variants at dysbindin associated with schizophrenia risk have been inconsistent across studies (20); the C-A-T risk haplotype in our sample is associated with reduced cortical expression of the gene, poorer educational attainment, and poorer spatial working memory $(2,8)$. Given these previous associations, we hypothesized that carriers of the risk haplotype would have a significantly attenuated P1 response compared with patients who were nonrisk carriers.

\section{Methods and Materials}

Subjects

Informed consent was obtained from 26 individuals who met DSM-IV criteria for schizophrenia (confirmed using the Struc- 
tured Clinical Interview for DSM [SCID]; [ 21]). These patients formed part of our larger genetics of psychosis sample, all of whom were of Irish origin (Irish parents and grandparents) and comprised 14 risk haplotype carriers and 12 nonrisk haplotype carriers. Criteria for inclusion in the study were that patients were in the view of their treatment team were clinically stable and 1) aged between 18 and 60 years, 2) had no history of substance abuse in the preceding 6 months, 3) had no other psychiatric diagnosis, 4) had no history of head injury with loss of consciousness, and 5) had no history of epilepsy or seizures. Because patients had already been genotyped for dysbindin, patients were re-recruited from the overall genetics sample on the basis of their risk haplotype status to ensure comparable numbers of risk and nonrisk carriers in the study.

Eleven of the risk haplotype carriers (mean age $=44.92$, SD $=$ 9.7), and 9 of the 12 nonrisk haplotype carriers (mean age 44.43, SD $=10.0$ ) were right-handed as assessed by the Edinburgh Handedness Inventory (22). All subjects reported normal or corrected-to-normal vision. No sex differences were apparent between groups; 11 (81\%) risk haplotype carriers were men versus $9(75 \%)$ nonrisk haplotype carriers; $\left.\chi^{2}=.56 ; p \geq .45\right)$. Duration ofi llness did not differ between groups (mean years for carriers was 25.1 [SD $=10.8$ ], and mean years for noncarriers was 22.8 [SD = 10.0]). When compared on the Positive and Negative Symptoms Severity Scale, no difference in symptom severity was observed between groups (mean positive symptoms scale score for carriers was 23.9 [SD $=9.5$ ] versus 24.8 [SD $=95$ ] for noncarriers, $\mathrm{t}(24)=-.67 ; \mathrm{p} \geq .5 ;$ mean negative symptoms scale scores for carriers was 18.4 [SD $=8.5$ ] versus 17.8 [SD $=8.0$ ] for noncarriers, $\mathrm{t}(24)=.56, \mathrm{p} \geq .50$ ). All patients were on psychotropic medication at the time of testing. Although dosage information was not available, there were no differences between groups in either type of antipsychotic prescribed (eight carriers [57\%] were prescribed atypical antipsychotics vs. six [52\%] noncarriers; $\chi^{2}=.31 ; p \geq .58$ ), number prescribed clozapine specifically (two carriers [14 3\%] vs. two [6.6\%] noncarriers; $\chi^{2}=$ $.31 ; p \geq .69)$, or number of patients prescribed anticholinergics (two carriers [15\%] vs. $1[10 \%]$ noncarrier; $\chi^{2}=.70 ; p \geq .40$ ). The lack of difference between carriers and noncarriers on each of variable (age, sex, duration of Ilness, symptom severity scores, and medication prescribed) accorded with the lack of differences observed in our total genetic sample for these variables ( $n=372$; data available on request).

\section{Stimuli and Presentation}

In each experimental block, subjects were presented with approximately 100 isolated-check images. Each image subtended $4^{\circ} \times 4^{\circ}$ of visual angle in total and consisted of an $8 \times 8$ array of isolated checks with each individual check subtending $.32 \times .32$ degrees of visual angle, colored gray on a white background (i.e., the luminance of the check was below that of the background, producing negative contrast) at $64 \%$ contrast. These were randomly interspersed with 40 line drawings of two kinds of animal $\left(2.4^{\circ}\right.$ wide $\times 1.8^{\circ}$ high) on a white background. The $64 \%$ contrast condition was chosen to stimulate both the magnocellular (M) and parvocellular (P) systems. A different animal pair was chosen for each block, from a possible 22 total pairs. The isolated-check stimuli and one pair of animal stimuli are shown in Figure 1. On average, subjects completed 14.1 (range 12-15) blocks, each lasting $3 \mathrm{~min}$. Stimuli were presented centrally on a cathode ray tube computer monitor in random order, with the monitor located $160 \mathrm{~cm}$ directly in front of the seated subject. (a)

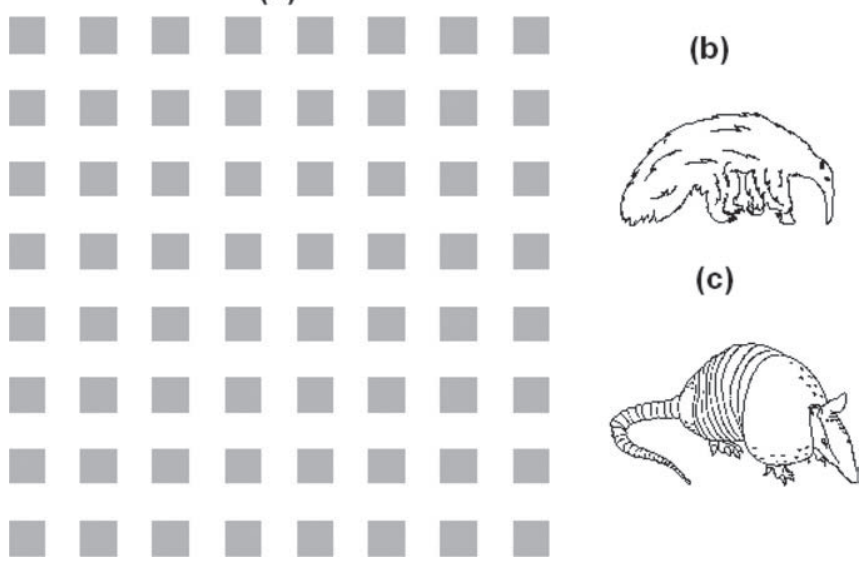

Figure 1. The centrally presented visual stimuli used in the task. Evenrelated potential waveforms were derived for the isolated check nontarget stimulus (a), whereas target discrimination was performed on the basis of infrequently presented animal line drawings ( $b$ and $c$ ).

The timing of the presentations was such that each image appeared for $60 \mathrm{msec}$ with a variable interstimulus interval (ISI) between 740 and $1540 \mathrm{msec}$ (randomly in steps of $200 \mathrm{msec}$ ) during which there was a blank white screen. The target animal was displayed at the start of the task, and subjects were asked to respond each time this animal was presented by pressing a button with their right thumb. They were told only to respond to target animals and to try to withhold responses to any other animal presented. The target and nontarget animals were presented with equal probability, ensuring that a subject could not rely on the exogenous alerting nature of any noncheckerboard stimulus to respond. Furthermore, the task of discrimination was made difficult by pairing similar-looking animals (e.g., dolphin and whale). The use of this task ensured that subjects were actively observing the stimuli. Only ERPs to the standard checkerboard stimuli were analyzed here.

Electrophysiological Data Acquisition and Statistical Analysis

Continuous electroencephalogram (EEG) was acquired through the ActiveTwo BioSemi electrode system (BioSemi, Amsterdam, the Netherlands) from 72 scalp electrodes, digitized at $512 \mathrm{~Hz}$ with an open passband from DC to $150 \mathrm{~Hz}$. (For a detailed description of the referencing and grounding conventions used by the BioSemi active electrode system, refer to http://www.biosemi.com/faq/cms\&drl.htm .) For analysis and display purposes, data were subsequently filtered with a 0 phase-shift $45-\mathrm{Hz}$ low-pass filter ( $24 \mathrm{~dB} /$ octave) after acquisition. No high-pass filter was applied. All data were re-referenced to a nasion electrode after acquisition, for analysis.

Data were analyzed using BESA Version 5.08 (Brain Electrical Source Analysis) software ( http://www.besa.de ; MEGIS Software $\mathrm{GmbH}$, Grafelfing, Germany). All electrode channels were subjected to an artifact criterion of $\pm 120 \mu \mathrm{V}$ from -200 to $400 \mathrm{msec}$ to reject trials with excessive electromyogram or other noise transients. The vertical and horizontal electrooculograms were also visually inspected for blinks and large eye movements, and affected epochs were then manually excluded. Epochs were calculated for a time-window from $200-\mathrm{msec}$ prestimulus to 400 -msec poststimulus, and baseline-corrected relative to the interval -200 to $0 \mathrm{msec}$. Accepted trials were then averaged only for the isolated-check stimuli. Only one subject had less than 700 
sweeps, but inspection of his data showed near zero noise levels, and thus it was deemed reasonable to include his data. The average epoch acceptance rate for the risk group was $62.7 \pm$ $12.2 \%$ (mean 1055 7; SD 200.6; range 802-1457), and for the nonrisk group $61.6 \pm 16.2 \%$ (mean 922; SD 24.1; range $418-$ 1347).

Our primary analysis was motivated by a specific hypothesis on the basis of previous research by our group regarding reduction in early visual sensory processing (i.e., the P1 processing period) in healthy first-degree relatives, relative to healthy control subjects. A measure of P1 amplitude was defined as the area under the curve (vs. the $0-\mu \mathrm{V}$ baseline) spanning the P1 component, found in the 80 - to $95-\mathrm{msec}$ interval, with a peak between 91 and $92 \mathrm{msec}$ (Figure 2A). These area measures were then submitted to a repeated-measures analysis of variance (ANOVA) using SPSS software (SPSS Inc., Chicago, Illinois) with between-subjects factor of group (dysbindin risk carriers versus noncarriers) and within-subjects factor of scalp region (left parietooccipital/right parietooccipital). P1 responses were averaged from six symmetrical pairs of scalp sites over parietooccipital scalp sites (P1/P3/P5/P7/PO7/PO3 for the left and P2/P4/P6/ $\mathrm{P} 8 / \mathrm{PO} 8 / \mathrm{PO} 4$ for the right). These electrodes were selected on the basis of previous evidence that they are positioned at the centre of the topographic distributions of the P1 component $(11,16)$. All tests were two-tailed with a preset alpha level of $p<$ .05. In all statistical tests, Greenhouse-Geisser Correction was used.

\section{Genotyping}

The dysbindin "risk" haplotype identified by Williams et al. (19) is derived from alleles C-A-T at single nucleotide polymorphisms (SNPs) rs2619539, rs3213207, and rs2619538. All SNPs were genotyped using Custom Taqman SNP Genotyping Assays from Applied Biosystems (Nattick, Massachusetts) on an $\mathrm{ABI}$ Prism 7900HT Sequence Detection System (Applied Biosystems). Duplicate genotyping in a subset of this sample indicated a genotyping error level of $<1 \%$. To compare dysbindin at-risk haplotype carriers (estimated frequency of 0.21 in cases in the overall sample) with noncarriers, assignment of haplotype carrier status to individual cases was performed using the Bayesian approach to reconstructing haplotypes implemented in the programme PHASE (v2. 0.2) ( 23). A case was only included in the study if the identity ofi ts two haplotypes could be determined at $p>.95$. Only $5 \%$ of patients in our sample were found to carry two copies of the C-A-T risk haplotype, and these individuals did not differ demographically or clinically from those carrying one copy of the haplotype. Therefore all analysis was carried out as a direct comparison between carriers and noncarriers of the risk haplotype. Individual SNPs (or alternative haplotypes) were not analyzed separately in relation to ERP data to minimize the issue of multiple testing.

\section{Results}

The mean target hit rate (to target animals) for nonrisk carriers was .854 (reaction time: $526.3 \mathrm{msec} ; \mathrm{SD}=56.9$ ), and for risk carriers was .882 (reaction time: 543.9 ; SD $=81.9$ ). The mean rates did not differ significantly between groups $[\mathrm{t}(24)=.488, \mathrm{p}$ $\geq$.63]. The mean correct rejection rate (to nontarget animals) for nonrisk subjects was .953 and .965 for risk subjects. The mean rates did not differ significantly between groups $[\mathrm{t}(24)=.810, \mathrm{p}$ $\geq .426$ ]. These rates indicate that subjects were actively engaged in this task and must have been centrally fixating to accurately identify target stimuli.

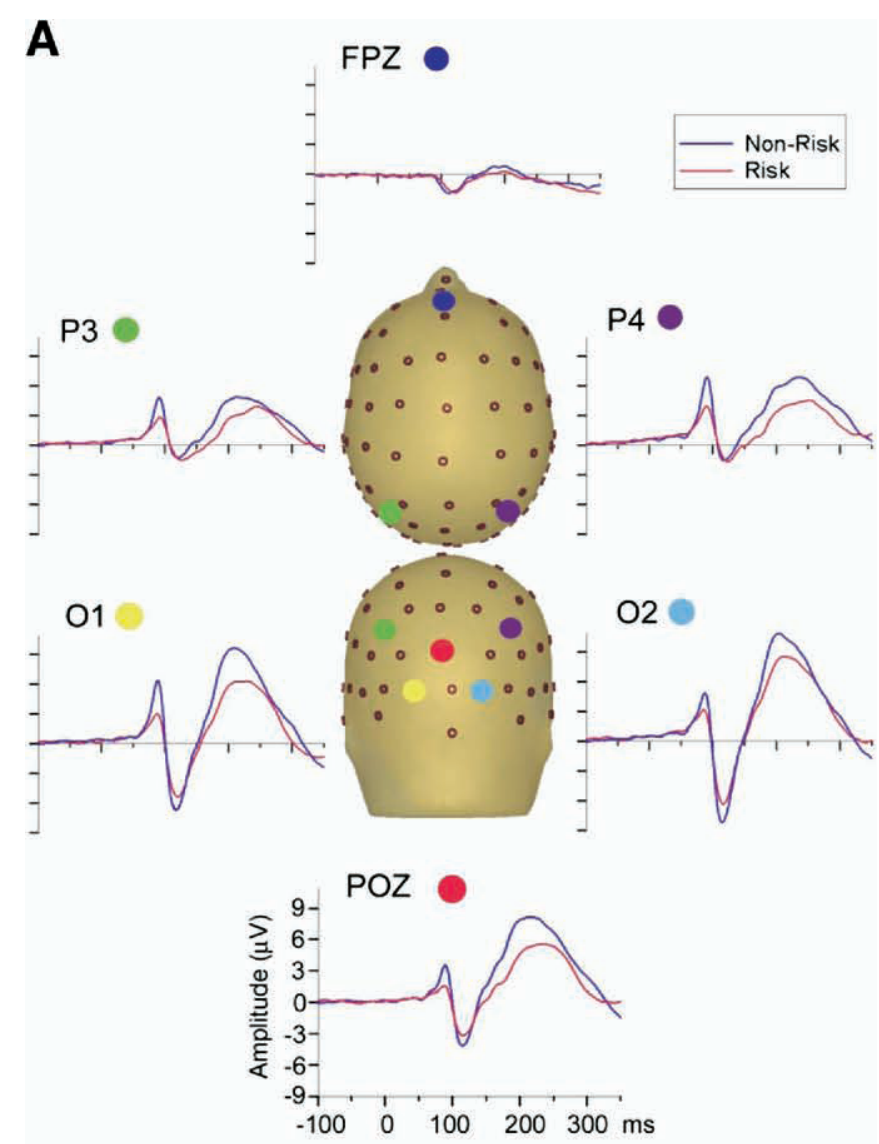

B

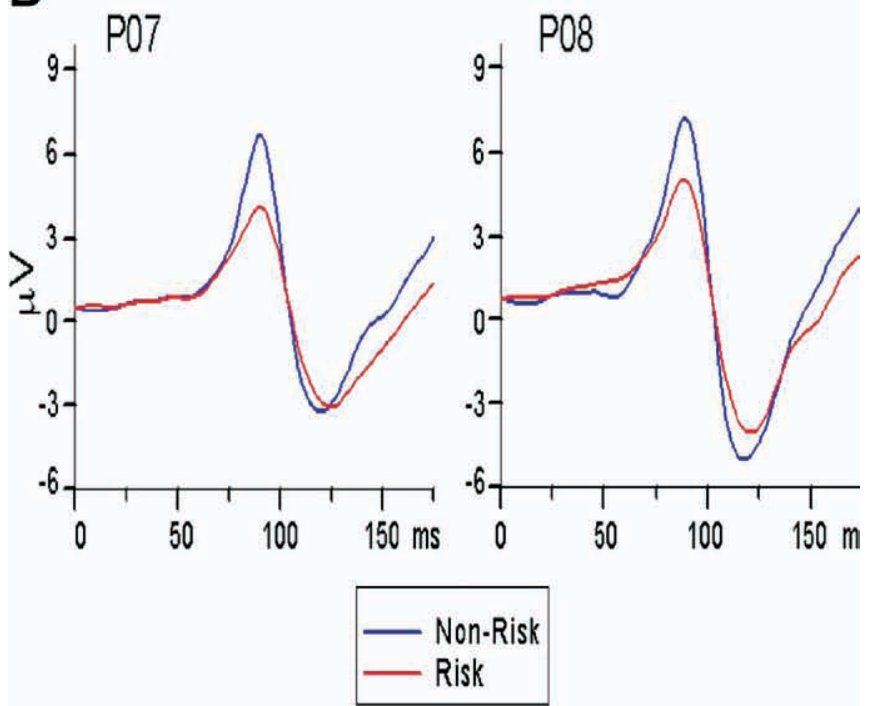

Figure 2. (A) Even-related potential morphology across the scalp for both groups illustrating responses from six representative electrodes spanning frontal, parietal, and occipital scalp regions. (B) P1 morphology, over a shorter timebase, for two additional parietooccipital scalp sites where the P1 difference was observed. Non-risk: non-carriers of the dysbindin risk haplotype; Risk: carriers of the dysbindin risk haplotype.

Figure 2A provides an overall illustration of ERP morphology across the scalp for both groups, showing responses from six representative electrodes spanning frontal, parietal, and occipital scalp regions. Group differences are clearly evident, particularly 


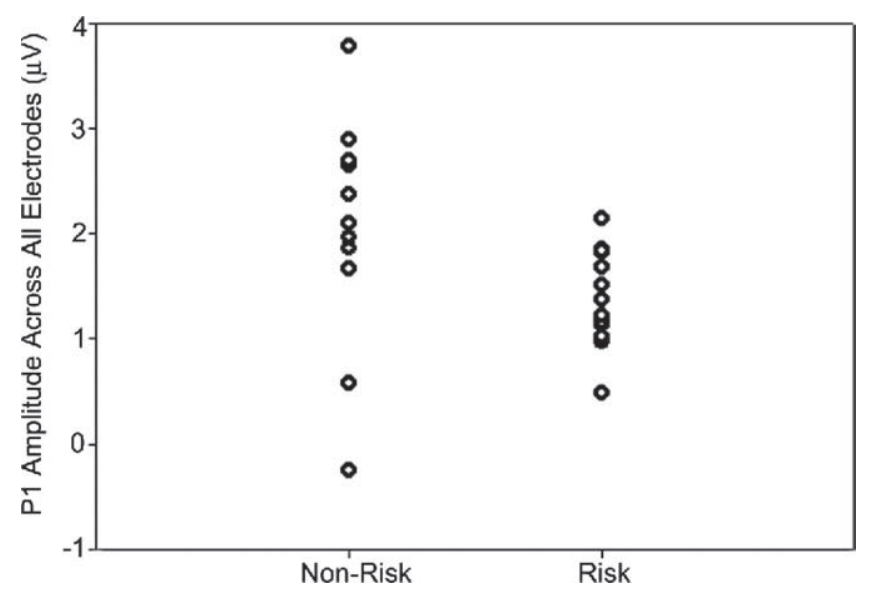

Figure 3. Scatterplot of P1 amplitudes (measured at its maximal point) for individual patient on the basis of multielectrode mean values from each electrode used in statistical analyses.

in posterior regions. Figure $2 \mathrm{~B}$ shows enlarged versions, on a shorter timebase, for two additional parietooccipital scalp sites where the P1 difference was observed. The distribution of P1 amplitudes for both groups at electrode site P6, the site at which the P1 difference was found to be largest, is shown in the form of a scatter plot in Figure 3. The mean P1 amplitudes were 3.54 $(\mathrm{SD}=.966)$ and $5.85(\mathrm{SD}=2.13)$ for risk haplotype carriers and noncarriers, respectively.

A $2 \times 2$ ANOVA ( 2 groups $\times 2$ scalp regions) was used to compare P1 amplitudes between groups over the left and right lateral occipital visual areas. We observed a significant main group effect, the $\mathrm{P} 1$ being of significantly smaller amplitude in the risk group $[F(1,24)=7.22, p=.013]$. A significant main effect of region was also present $[F(1,24)=5.40, p=.029]$, indicating that the P1 was generally larger over the right than the left hemiscalp. There was no interaction of the factors Group and Region $[F(1,24)=256, p=.618]$. An effect size of $d=.89$ was calculated for the difference in P1 amplitude measured between groups over the interval $80-95 \mathrm{msec}$, which constitutes a large effect size according to the criterion of Cohen. Figure 4 shows mapping of the difference topography associated with dysbindin.

Following the observation of robust P1 differences between dysbindin carriers and noncarriers, differences between groups during the N1 component were also analyzed. The Visual N1 component was seen to peak at $120 \mathrm{msec}$ for both groups, and it had a distinct midline inferior occipital topography. It was clearly maximal over electrodes $\mathrm{O} 1, \mathrm{OZ}, \mathrm{O} 2$, and IZ. To test for significant group differences, an integrated amplitude measure was derived for the 120-130 msec time window spanning the peak at these four electrode sites. These values were then averaged and submitted to a simple two-tailed t test. No group difference in $\mathrm{N} 1$ amplitude was found $[\mathrm{t}(2,24)=-.18, \mathrm{p}=.85)$.

\section{Discussion}

Impaired P1 generation has repeatedly been demonstrated in patients with schizophrenia (10-15) and recently in their firstdegree relatives, supporting a genetic contribution to this deficit (16). We investigated the relationship between early visual processing deficits as indexed by the P1 and a dysbindin haplotype associated with increased schizophrenia risk and poorer higher cognitive functioning in our sample. We observed that patients with schizophrenia who carried the dysbindin risk haplotype showed significantly greater P1 deficits than patients with schizophrenia who were noncarriers. The observed decrement in P1 generation was found in the absence of differences between groups on age, gender, duration ofi llness, symptoms severity, or medication effects. The observed difference between risk carriers and noncarriers is not subtle. In scalp sites where the effect was largest, risk haplotype carriers showed maximal P1 amplitudes that were little more than half those seen in patients who were noncarriers, despite both groups having similar illness duration. The parietooccipital location of this deficit accords closely with our previous studies in chronic patients $(10,11,14)$.

How risk variants at dysbindin affect gene function is uncertain, but the risk haplotype investigated in this study is of particular interest because it is associated with reduced cortical expression of dysbindin (2). Variants in dysbindin that are associated with schizophrenia risk have been identified as associated with poorer general cognitive performance in controls (6) and with both general and specific cognitive deficits in patients with schizophrenia $(6,8)$, although the variants identified have been inconsistent across groups (20). Our study extends these previous reports by indicating that variation at the gene may play a significant role in early visual processing as well as higher cognitive processing. The influence of dysbindin on multiple cognitive and sensory processes both in patients and control subjects suggests a more general role for dysbindin in brain function, rather than a regionally or cognitively specific role. As with most brain-expressed genes, dysbindin is widely expressed and lacks regional specificity ( http://www.brain-map.org ) (2-4), and we view this as consistent with a global involvement in presynaptic glutamate availability. This view of dysbindin's more general impact on brain behavior is further supported by recent evidence ofi ts association with increased negative symptom severity (24-25), symptoms that co-occur with, but are only partly explained by, cognitive deficits.

As an endophenotype measure, the P1 has the major advantage of being relatively easy to measure quickly and accurately. As a largely automatic response, it is not as susceptible to the same motivational factors or fluctuations in clinical state as later cognitive components such as the P300. However, this is not to say that the P1 is not cognitively penetrable. Although early stages of perceptual processing (from as early as $50-100 \mathrm{msec}$ poststimulus) serve an important role in "spotlighting" of relevant

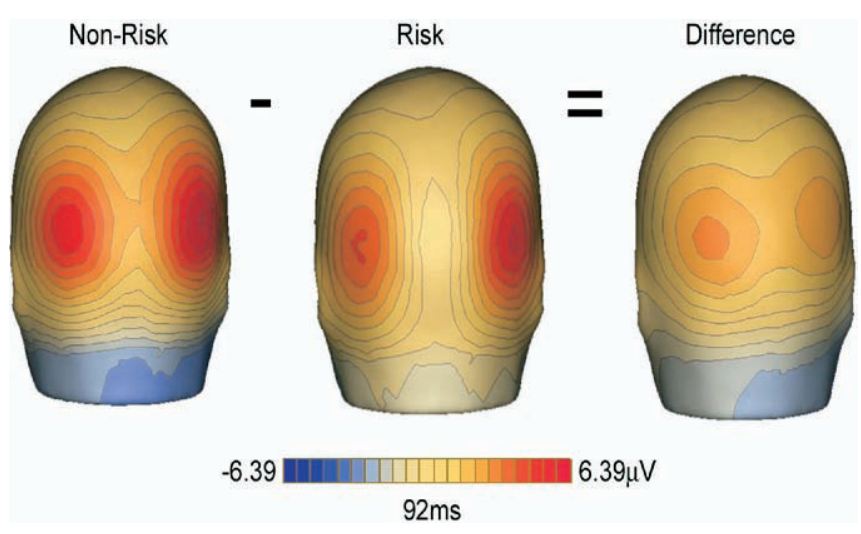

Figure 4. Mapping of the difference topography associated with dysbindin. Subtraction of the grand-averaged waveforms of the risk group from those of the nonrisk group enables the difference effect to be isolated and mapped. The P1 decrement has a bilateral lateral occipital distribution, not dissimilar to that of the base maps of the P1. 
information for later processing, these early processing stages (from 70 msec onward) appear to be reciprocally modulated by higher processing areas (26). It is therefore interesting that in our sample, a twin association between a dysbindin risk haplotype and both sensory and higher cognitive processes is evidenced (previously) by its association with poorer visuospatial working memory (8) and in this study by its association with early visual processing deficits. These associations may reflect the reciprocal relationship between early sensory and higher cognitive function, particularly for visual information. On one hand, deficits in "capturing" visual information are likely to increase difficulties in efficiently maintaining and updating that information "online" during spatial working memory tasks. Conversely, an inability to maintain context during later stages of context processing leads to difficulties focusing on relevant information during earlier stages of visual processing. Although the purpose of our study was not to tease apart the relationship between P1 deficits and attentional control, the notion that the P1 deficits we observed are a result of spatial attention deficits is highly unlikely. The equivalent performance of both groups in target detection rates suggests that the groups did not differ in fixation on and attention to the centrally presented stimuli (although it cannot be inferred that the groups were of equivalent cognitive ability, given the simplicity of the task used). Furthermore, the eliciting of the P1 by centrally presenting stimuli is not modulated by directing attention to or from that location (unlike peripheral stimuli), again making this effect unlikely to result from deployment of visual spatial attention (27). In either case, this study evidences a shared genetic component in both processes wherein the deleterious effects of dysbindin are seen in the same sample at both sensory and higher cognitive levels of processing.

The effects of dysbindin on early sensory processing in this relatively small sample will require replication in independent case samples to establish its true effect size, particularly for the other identified dysbindin risk variants (20). Such replication is important to establish the predictive value of dysbindin in delineating a schizophrenia subgroup with pronounced sensorylevel visual processing deficits. In turn, this predictive ability would contribute to establishing the utility of P1 deficits as a potential biomarker for investigating dysbindin and related schizophrenia susceptibility genes. Follow-up study of these effects in both relatives and unaffected control subjects genotyped for this risk haplotype will determine whether this effect interacts with disease risk (genotype information was not available to investigate this in relatives and control subjects who participated in our previous studies). Given the difference in associated alleles and haplotypes associated schizophrenia across studies, whether other dysbindin variants associated with schizophrenia are associated with sensory processing also merits investigation. Finally, using a larger sample of patients and control subjects the investigation of dysbindin's association with early visual deficits could be extended to investigate whether these deficits correlate with the behavioral deficits already described in this group but that we did not have power to investigate in our EEG sample.

\section{Conclusions}

Early visual processing occurs via a glutamate dependent brain system $(28,29)$. Both visual processing deficits and glutamate system abnormalities are increasingly implicated in schizophrenia. A role for presynaptic dysbindin in this transmitter system has already been highlighted $(3,5)$. The reduced P1 VEP associated with the dysbindin risk haplotype previously identi- fied in our sample presents functional confirmation ofi ts deleterious effect on brain activity, making it likely to be part of the neurobiology of schizophrenia.

Financial support for this study was provided by grants from the National Institute of Mental Health (NIMH) in the United States (Grant Nos. MH65350 to JJF and MH49334 to DCJ), the Science Foundation Ireland (MG and $A C$ ), and Higher Education Authority Ireland (MG and AC).

None of the authors have conflicts ofi nterests to declare in relation to this study.

We thank Dr. Sherlyn Yeap for help facilitating patient recordings at the Cognitive Neurophysiology Laboratory at St. Vincent's Hospital in Fairview, Dublin.

1. Norton N, Williams HJ, Owen MJ (2004): An update on the genetics of schizophrenia. Curr Opin Psychiatry19:158-164.

2. Bray NJ, Preece A, Williams NM, Moskvina V, Buckland PR, Owen MJ, et al. (2005): Haplotypes at the dystrobrevin binding protein 1 (DTNBP1) gene locus mediate risk for schizophrenia through reduced DTNBP1 expression. Hum Mol Genet14:1947-1954.

3. Talbot K, Eidem WL, Tinsley CL, Benson MA, Thompson EW, Smith RJ (2004): dysbindin-1 is reduced in intrinsic, glutamatergic terminals of the hippocampal formation in schizophrenia.J Clin Invest113:1353-1363.

4. Weickert CS, Straub RE, McClintock BW, Matsumoto M, Hashimoto R, Hyde TM, et al. (2004): Human dysbindin (DTNBP1) gene expression in normal brain and in schizophrenic prefrontal cortex and midbrain. Arch Gen Psychiatry61:544-555.

5. Numakawa T, Yagasaki Y, Ishimoto T, Okada T, Suzuki T, Iwata N, et al. (2004): Evidence of novel neuronal functions of dysbindin, a susceptibility gene for schizophrenia. Hum Mol Genet13: 2699-2708.

6. Burdick KE, Lencz T, Funke B, Finn CT, Szeszko PR, Kane JM, et al. (2006): Genetic variation in DTNBP1 influences general cognitive ability. Hum Mol Genet15:1563-1568.

7. Fallgatter AJ, Herrmann MJ, HohoffC, Ehlis AC, Jarczok TA, Freitag CM, et al. (2006): DTNBP1 (dysbindin) gene variants modulate prefrontal brain function in healthy individuals. Neuropsychopharmacology 31:2002-2010.

8. Donohoe G, Morris DW, Clarke S, McGhee KA, Schwaiger S, Nangle JM, et al. (2007): Variance in neurocognitive performance is associated with dysbindin-1 in schizophrenia: A preliminary study. Neuropsychologia 45:454-458.

9. Butler PD, Javitt DC (2005). Early-stage visual processing deficits in schizophrenia. Curr Opin Psychiatry18:151-157.

10. Foxe JJ, Doniger GM, Javitt DC (2001): Early visual processing deficits in schizophrenia: Impaired P1 generation revealed by high-density electrical mapping. Neuroreport12: 3815-3820.

11. Foxe JJ, Murray MM, Javitt DC (2005): Filling-in in schizophrenia: a highdensity electrical mapping and source-analysis investigation of llusory contour processing. Cereb Cortex15:1914-1927.

12. Butler PD, Schechter I, Zemon V, Schwartz SG, Greenstein VC, Gordon J, et al. (2001): Dysfunction of early-stage visual processing in schizophrenia. Am J Psychiatry158:1126-1133.

13. Butler PD, Zemon V, Schechter I, Saperstein AM, Hoptman MJ, Lim KO, et al. (2005): Early-stage visual processing and cortical amplification deficits in schizophrenia. Arch Gen Psychiatry62:495-504.

14. Doniger GM, Foxe JJ, Murray MM, Higgins BA, Javitt DC (2002): Impaired visual object recognition and dorsal/ventral stream interaction in schizophrenia. Arch Gen Psychiatry59:1011-1020.

15. Schechter I, Butler PD, Zemon VM, Revheim N, Saperstein AM, Jalbrzikowski M, et al. (2005): Impairments in generation of early-stage transient visual evoked potentials to magno- and parvocellular-selective stimuli in schizophrenia. Clin Neurophysiol1 16:2204-2215.

16. Yeap S, Kelly SP, Sehatpour P, Magno E, Javitt DC, Garavan H, et al. (2006): Early visual sensory deficits as endophenotypes for schizophrenia: High-density electrical mapping in clinically unaffected first-degree relatives. Arch Gen Psychiatry63:1180-1188.

17. Liu 2004 Liu Z, Tam WC, Xue Z, Yao S, Wu D (2004): Positive and negative symptom profile schizophrenia and abnormalities in the P300 component of the event-related potential: A longitudinal controlled study. Psychiatry Res132:131-139. 
18. Wood SM, Potts GF, Hall JF, Ulanday JB, Netsiri C (2006): Event-related potentials to auditory and visual selective attention in schizophrenia. Int J Psychophysiol 60:67-75.

19. Williams NM, Preece A, Morris DW, Spurlock G, Bray NJ, Stephens M, et al. (2004): Identification in 2 independent samples of a novel schizophrenia risk haplotype of the dystrobrevin binding protein gene (DTNBP1). Arch Gen Psychiatry61:336-344.

20. Mutsuddi M, Morris DW, Waggoner SG, Daly MJ, Scolnick EM, Sklar P (2006): Analysis of high-resolution HapMap of DTNBP1 (dysbindin) suggests no consistency between reported common variant associations and schizophrenia. Am J Hum Genet79:903-909.

21. American Psychiatric Association (1992): Structured Clinical Assessment for the DSM-III-R Washington, DC: American Psychiatric Association.

22. Oldfield RC (1971): The assessment of handedness: The Edinburgh inventory. Neuropsychologia9:97-113.

23. Stephens M, Donnelly P (2003): A comparison of bayesian methods for haplotype reconstruction from population genotype data. Am J Hum Genet73:1162-1169.
24. Fanous $A H$, van den Oord EJ, Riley BP, Aggen SH, Neale MC, O'Neill FA, et al. (2005): Relationship between a high-risk haplotype in the DTNBP1 (dysbindin) gene and clinical features of schizophrenia. Am J Psychiatry 162:1824-1832.

25. DeRosse P, Funke B, Burdick KE, Lencz T, Ekholm JM, Kane JM, et al. (2006): dysbindin genotype and negative symptoms in schizophrenia. Am J Psychiatry. 163:532-534.

26. Martinez A, Anllo-Vento L, Sereno MI, Frank LR, Buxton RB, Dubowitz DJ (1999): Involvement of striate and extrastriate visual cortical areas in spatial attention. Nat Neurosci2: 364-369.

27. Handy TC, Khoe W (2005): Attention and sensory gain control: A peripheral visual process? J Cogn Neurosci17:1936-1949.

28. Kwon YH, Nelson SB, Toth LJ, Sur M (1992): Effect of stimulus contrast and size on NMDA receptor activity in cat lateral geniculate nucleus. J Neurophysiol68:182-196.

29. Fox K, Daw N, Sato H, Czepita D (1992): The effect of visual experience on development of NMDA receptor synaptic transmission in kitten visual cortex. J Neurosci12:2672-2684. 